\title{
Tables of Nuclear Cross Sections and Reaction Rates: an Addendum to the Paper "Astrophysical Reaction Rates from Statistical Model Calculations"
}

\author{
Thomas Rauscher and Friedrich-Karl Thielemann
}

Departement für Physik und Astronomie, Universität Basel, Klingelbergstr. 82, CH-4056 Basel, Schweiz (Switzerland)

\begin{abstract}
In a previous publication [Atomic Data and Nuclear Data Tables 75, 1 (2000)], we had given seven parameter analytical fits to theoretical reaction rates derived from nuclear cross sections calculated in the statistical model (Hauser-Feshbach formalism) for targets with $10 \leq Z \leq 83$ (Ne to $\mathrm{Bi}$ ) and for a mass range reaching the neutron and proton driplines. Reactions considered were $(\mathrm{n}, \gamma),(\mathrm{n}, \mathrm{p}),(\mathrm{n}, \alpha),(\mathrm{p}, \gamma),(\mathrm{p}, \alpha),(\alpha, \gamma)$, and their inverse reactions. Here, we present the theoretical nuclear cross sections and astrophysical reaction rates from which those rate fits were derived, and we provide these data as on-line electronic files. Corresponding to the fitted rates, two complete data sets are provided, one of which includes a phenomenological treatment of shell quenching for neutron-rich nuclei.
\end{abstract}

\section{Contents}

\begin{tabular}{lll}
\hline & Introduction & 2
\end{tabular}

2 Tabulated information 3

2.1 Laboratory Cross Sections For Capture Reactions and Exother-

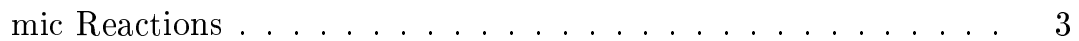

2.1.1 Instructions for the Use of the Cross Section Files . . . . 5

2.2 Astrophysical Reaction Rates For Capture Reactions and Exothermic Reactions ...................... 6

2.2.1 Instructions for the Use of the Rate Files . . . . . . . . . 6

$\begin{array}{lll}2.3 & \text { Reverse Stellar Rates and Endothermic Laboratory Cross Sections } 7\end{array}$

2.4 Experimental Nuclear Level Schemes . . . . . . . . . . 8

2.4.1 Instructions for the Use of the Nuclear Level File . . . . . 8

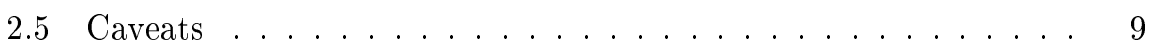


2.5.1 Applicability of the Statistical Model . . . . . . . . . . . . 9

2.5.2 Validity of the Predictions . . . . . . . . . . . . . . . 9

$\begin{array}{lll}3 & \text { Summary } & 10\end{array}$

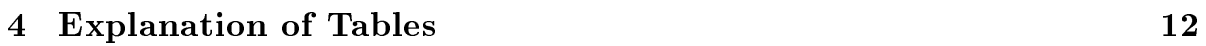

Table I: Sample of Cross Section Tables 15

Table II: Sample of Rate Tables 20

Table III: Sample of Nuclear Level Table 23

\section{Introduction}

In a previous paper by Rauscher and Thielemann (価), hereafter referred to as RATH) we presented fits to nuclear reaction rates covering a wide range of isotopes and most of the nuclear chart. The data sets of reaction rate fits in that paper were mainly suited to direct application in astrophysical simulations. However, in certain computational approaches it is preferable to have the rates in the original tabular format without any further fitting procedure which, on one hand, may be advantageous for the speedy calculation of a rate at a given temperature with an economical use of computer memory but, on the other hand, may introduce additional inaccuracies. Furthermore, for nuclear physics applications it is often preferable to have the tables of nuclear cross sections directly. Therefore we thought it worthwhile to also publish the original cross sections and reaction rates from which the fits given in RATH have been derived; we provide these extensive data in the form of electronic tables. (It should be noted that "data" in this context refers to results of theoretical calculations; wherever there is a reference to experimental data, it is stated explicitly.)

As in RATH, we provide here two sets of calculations with input from two different mass models, the finite range droplet model (FRDM) [2] and an extended Thomas-Fermi approach with Strutinski integral (ETFSI-Q) [3]. Experimental masses and level properties had been used in the calculations where available. In addition to cross sections and reaction rates, particle separation energies are given. In order to facilitate a comparison to other calculations, all the experimental level information used in our calculations is shown in a separate table.

Here, we do not want to repeat the detailed definitions of the statistical model and of reaction rates already given in RATH. Therefore we limit ourselves to the most concise form and refer the reader to RATH for more details. In the following, we concentrate on the explanation of how to extract values from the on-line tables and on giving a few caveats when using the tabulated information 


\section{Tabulated information}

The data set consists of seven tables provided as separate on-line files. The four files described in Secs. 2.1 and 2.2 contain the raw data used to generate the fits given in RATH. Further information which may be useful for comparison to experiments or other calculations is given in three additional files (see Secs. 2.3 and 2.4). All files are formatted in such a way as to be machine readable without losing the accessibility to the human reader. That way, the additional information provided here can best serve the needs of several specialized fields of nuclear physics as well as astrophysics. Definitions of cross sections and reaction rates as well as further details of the statistical model calculations are already given in RATH.

\subsection{Laboratory Cross Sections For Capture Reactions and Exothermic Reactions}

Reaction cross sections involving neutrons, protons and $\alpha$ particles as projectiles or ejectiles have been calculated for targets from Ne up to, and including, Bi. The results of two different sets of calculations are given here in two files. They differ in the mass model used, which enters into the computation of the separation energies and $Q$ values as well as into the microscopic input to the level density calculation. One set was calculated employing the well-known FRDM mass model [2], the other set employed the ETFSI-Q model [3] which includes a phenomenological approximation of shell-quenching effects (see RATH for further details on the mass models).

Tabulated are the laboratory cross sections for capture reactions and for exoergic particle reactions. These assume that the target is in the ground state and cannot, in general, be used for computation of an astrophysical rate as given in RATH (cf. Eqs. (1), (2), (10) in RATH). However, they can be directly compared to experimentally measured data. The isotope range is as specified in Table A of RATH, which is repeated here again for convenience. Cross sections for exothermic $(Q \geq 0)$ reactions with particles in the exit channel are given here as well as all capture reactions regardless of the $Q$ value. All those reactions are contained in one file each for each mass model, sorted by target (charge and mass number) and projectile (neutron, proton, $\alpha$ particle), in that order. The energy grid is different for each combination of target and projectile as it was chosen in such a way as to provide the best grid for the numerical integration involved in the computation of the astrophysical reaction rate. This means that, e.g., the energies are densely clustered around channel openings and more widely spaced in regions of "well-behaved" cross sections. The energies are given in $\mathrm{MeV}$; the cross sections are given in barn $\left(1\right.$ barn $\left.=10^{-28} \mathrm{~m}^{2}=100 \mathrm{fm}^{2}\right)$.

Provided as additional information are the particle separation energies in the compound nucleus, from which the reaction $Q$ values given in RATH were derived. 
TABLE A

Isotope Range of the On-line Cross Section and Reaction Rate Files

\begin{tabular}{|c|c|c|c|c|c|c|c|c|c|}
\hline \multirow[b]{2}{*}{$Z$} & \multicolumn{2}{|c|}{ FRDM } & \multicolumn{2}{|c|}{ ETFSI-Q } & \multirow[b]{2}{*}{$Z$} & \multicolumn{2}{|c|}{ FRDM } & \multicolumn{2}{|c|}{ ETFSI-Q } \\
\hline & $N_{\min }$ & $N_{\max }$ & $N_{\min }$ & $N_{\max }$ & & $N_{\text {min }}$ & $N_{\text {max }}$ & $N_{\min }$ & $N_{\text {max }}$ \\
\hline 8 & 5 & 10 & & & 47 & 41 & 113 & 41 & 111 \\
\hline 9 & 5 & 28 & & & 48 & 42 & 115 & 42 & 112 \\
\hline 10 & 5 & 31 & & & 49 & 43 & 117 & 43 & 113 \\
\hline 11 & 6 & 33 & & & 50 & 44 & 119 & 44 & 114 \\
\hline 12 & 7 & 35 & & & 51 & 46 & 121 & 46 & 115 \\
\hline 13 & 8 & 38 & & & 52 & 47 & 124 & 47 & 124 \\
\hline 14 & 8 & 40 & & & 53 & 48 & 126 & 48 & 126 \\
\hline 15 & 8 & 42 & & & 54 & 49 & 128 & 49 & 128 \\
\hline 16 & 8 & 44 & & & 55 & 51 & 130 & 51 & 130 \\
\hline 17 & 9 & 46 & & & 56 & 52 & 133 & 52 & 132 \\
\hline 18 & 9 & 49 & & & 57 & 53 & 135 & 53 & 133 \\
\hline 19 & 10 & 51 & & & 58 & 55 & 137 & 55 & 134 \\
\hline 20 & 10 & 53 & & & 59 & 56 & 139 & 56 & 135 \\
\hline 21 & 11 & 55 & & & 60 & 58 & 141 & 58 & 136 \\
\hline 22 & 12 & 58 & & & 61 & 59 & 144 & 59 & 137 \\
\hline 23 & 13 & 60 & & & 62 & 61 & 146 & 61 & 138 \\
\hline 24 & 14 & 62 & 18 & 62 & 63 & 62 & 148 & 62 & 139 \\
\hline 25 & 15 & 64 & 18 & 64 & 64 & 64 & 150 & 64 & 150 \\
\hline 26 & 16 & 66 & 19 & 66 & 65 & 65 & 153 & 65 & 152 \\
\hline 27 & 17 & 69 & 19 & 67 & 66 & 67 & 155 & 67 & 154 \\
\hline 28 & 18 & 71 & 20 & 68 & 67 & 69 & 157 & 69 & 155 \\
\hline 29 & 19 & 73 & 21 & 69 & 68 & 70 & 159 & 70 & 156 \\
\hline 30 & 21 & 75 & 22 & 70 & 69 & 72 & 161 & 72 & 157 \\
\hline 31 & 22 & 77 & 23 & 71 & 70 & 73 & 164 & 73 & 158 \\
\hline 32 & 23 & 80 & 24 & 72 & 71 & 75 & 166 & 75 & 159 \\
\hline 33 & 24 & 82 & 25 & 73 & 72 & 77 & 168 & 77 & 160 \\
\hline 34 & 25 & 84 & 26 & 84 & 73 & 78 & 170 & 78 & 161 \\
\hline 35 & 26 & 86 & 27 & 86 & 74 & 80 & 173 & 80 & 162 \\
\hline 36 & 27 & 88 & 28 & 88 & 75 & 81 & 175 & 81 & 163 \\
\hline 37 & 29 & 91 & 29 & 89 & 76 & 83 & 177 & 83 & 177 \\
\hline 38 & 30 & 93 & 30 & 90 & 77 & 85 & 179 & 85 & 179 \\
\hline 39 & 31 & 95 & 31 & 91 & 78 & 87 & 182 & 87 & 182 \\
\hline 40 & 32 & 97 & 32 & 97 & 79 & 88 & 184 & 88 & 184 \\
\hline 41 & 33 & 99 & 33 & 99 & 80 & 90 & 186 & 90 & 186 \\
\hline 42 & 35 & 102 & 35 & 102 & 81 & 92 & 188 & 92 & 188 \\
\hline 43 & 36 & 104 & 36 & 104 & 82 & 93 & 191 & 93 & 191 \\
\hline 44 & 37 & 106 & 37 & 106 & 83 & 95 & 193 & 95 & 193 \\
\hline 45 & 38 & 108 & 38 & 108 & 84 & 98 & 193 & 98 & 193 \\
\hline 46 & 40 & 110 & 40 & 110 & 85 & 101 & 195 & 101 & 195 \\
\hline
\end{tabular}

Note. Given are the charge number $Z$ and the lower and upper limits $N_{\min }$ and $N_{\max }$ of the neutron number in the isotopic chain. The complete files $c s$ frdm.asc, cs_etfsiq.asc, cs_frdm_endo.asc, cs_etfsiq_endo.asc, rates_frdm.asc, rates_etfsiq.asc, and levels.asc are available electronically. 


\subsubsection{Instructions for the Use of the Cross Section Files}

The file can either be browsed by using a text editing program or a code specifically written for reading the provided data. Table I shows an example of a segment from the FRDM file. The data blocks are sorted first by target nucleus (charge, mass number) and then by projectile. For each target-projectile combination, the capture cross sections are listed for all calculated projectile energies regardless of $Q$ value. Additionally, cross sections are given for those reactions with particles in the exit channel, having $Q \geq 0$. Thus, each data block will have a different number of lines, depending on the number of calculated energies and exoergic reaction channels. The lines within each block are organized as follows. Each data block for a specific combination of target and projectile starts with an identifying line specifying, in that order: charge and mass number of the target; a digit specifying the projectile ( 0 for neutron, 1 for proton, 2 for $\alpha$ particle); the number $L$ of calculated energies; indices $b$ and $c$ indicating whether or not a reaction with particles in the exit channel has $Q \geq 0$ and is therefore listed $(=1)$ or not listed $(=0)$; charge $Z c$ and mass number $A c$ of the compound nucleus; and the particle separation energies $S_{n}, S_{p}, S_{\alpha}$ of the compound nucleus. The end of the line gives again target isotope $(T$, projectile $(x)$ and the three possible exit channels denoted by roman letters $(\mathrm{g}=\gamma, \mathrm{n}, \mathrm{p}$, and $\mathrm{a}=\alpha$ ). Following the first line are the $L$ center-of-mass energies in $\mathrm{MeV}$ at which the cross sections were calculated. There are at most eight entries per line. Then, $L$ capture cross sections are printed, regardless of the $Q$ value. Values for the other reaction channels are only given if $Q \geq 0$. If $b=0$ and/or $c=0$, then no cross section values are printed for the respective exit channel 1,2 . Thus, the capture cross sections are followed by $L \times(b+c)$ cross sections and therefore a total of $L \times(1+b+c)$ cross sections is given. A new data block starts with the next identification line after the last line of cross sections.

As an example we consider the reaction ${ }^{70} \mathrm{Ga}(\mathrm{p}, \mathrm{n}){ }^{70} \mathrm{Ge}$. Since $Q=S_{p}\left({ }^{71} \mathrm{Ge}\right)-$ $S_{n}\left({ }^{71} \mathrm{Ge}\right)>0$ the cross sections can be found in the regular cross section file. Table I shows a small part of that table. Looking for ${ }^{70} \mathrm{Ga}$, e.g. by a text search for "ga70", one first encounters the data block for ${ }^{70} \mathrm{Ga}+\mathrm{n}$. Farther down one finds the identification line for ${ }^{70} \mathrm{Ga}+\mathrm{p}$ since for the same target isotope the data blocks are sorted by reaction. With a computer code it would be more economical to search for the numeric codes for charge, mass number and projectile at the beginning of the line. The relevant identification line tells us that the cross sections were calculated at 61 energies and that both the values for the $(p, n)$ as well as the $(p, \alpha)$ reaction are printed. This line is followed by the 61 center-of-mass energies, then 61 capture cross sections, $61(\mathrm{p}, \mathrm{n})$ cross sections and, finally, $61(\mathrm{p}, \alpha)$ cross sections. Thus, for instance, the value of the ${ }^{70} \mathrm{Ga}(\mathrm{p}, \mathrm{n}){ }^{70} \mathrm{Ge}$ cross section at $2.3022 \mathrm{MeV}$ is given as 2.964 millibarn. The RATH tables should always be consulted to determine if the statistical model is suited for predicting cross sections for a specific reaction at a given rate (see Sec. 2.5.1).

The values for the inverse reaction ${ }^{70} \mathrm{Ge}(\mathrm{n}, \mathrm{p}){ }^{70} \mathrm{Ga}$ cannot be extracted from the same file as $Q<0$ for this reaction. An inspection of the identification line 
for ${ }^{70} \mathrm{Ge}+\mathrm{n}$ farther down shows that $b=0$ and that therefore no cross sections are listed for this reaction. Particle reactions with $Q<0$ are given in a separate file, see Sec. 2.3.

The above instructions are relevant to the posted cross section files $c s \_f r d m . a s c$, cs_etfsiq.asc, cs_frdm_endo.asc, and cs_etfsiq_endo.asc.

\subsection{Astrophysical Reaction Rates For Capture Reactions and Exothermic Reactions}

Reaction rates are given in two separate files, one for each mass model, for the reactions as included in the cross section listings. The rates were computed on a grid of 24 temperatures: $T_{9}=0.1,0.15,0.2,0.3,0.4,0.5,0.6,0.7,0.8,0.9$, $1.0,1.5,2.0,2.5,3.0,3.5,4.0,4.5,5.0,6.0,7.0,8.0,9.0,10.0\left(T_{9}\right.$ is given in $10^{9} \mathrm{~K}$ ). The reactions are sorted as before. For each reaction, the laboratory rate $N_{A}\langle\sigma v\rangle^{\mathrm{lab}}$ as well as the stellar rate $N_{A}\langle\sigma v\rangle^{*}$ is given. The laboratory rate was computed directly from the cross sections discussed in Sec. 2.1 and can be used for comparison with rates derived from experiment. Only the stellar rate considers the thermal excitation in a stellar plasma and should be used exclusively in astrophysical calculations. It was computed making use of the stellar transmission functions (see Eqs. (2) and (3) in RATH). The stellar reaction rates were the basis for the RATH fits. The rates are given in units of $\mathrm{cm}^{3} \mathrm{~s}^{-1} \mathrm{~mol}^{-1}$.

\subsubsection{Instructions for the Use of the Rate Files}

An example for a segment of the FRDM rate file is given in Table II. The structure of the table is somewhat similar to the cross section table as described in Sec. 2.1.1. The data blocks are sorted first by target nucleus (charge, mass number) and then by projectile. For each target and projectile, the capture rates are printed, regardless of $Q$ value. The rates are also printed for those particle exit channels for which $Q \geq 0$. Thus, each data block will have a different number of total lines, depending on the number of exoergic particle channels. A data block starts with an identification line giving charge and mass number of target, the projectile ( 0 for neutron, 1 for proton, 2 for $\alpha$ particle), and indices $b$ and $c$ specifying whether a particle exit channel is listed. The digits $b$ and $c$ can only assume the values 0 (not listed) or 1 (listed). The end of the line gives again target isotope, projectile and the three possible exit channels. This is followed by 24 laboratory rates (three lines with eight entries each) calculated at the temperatures given in the previous section. A blank line separates the last line of the laboratory rates from the following three lines with stellar rates. Laboratory and stellar rates are given for the capture reaction regardless of the $Q$ value. This is followed by the laboratory and stellar rates of the particle exit channels for which $b=1$ and/or $c=1$, i.e. with $Q \geq 0$. Thus, there are $2 * 24 *(1+b+c)$ rates printed in total.

As an example, the reaction ${ }^{70} \mathrm{Ga}(\mathrm{p}, \mathrm{n}){ }^{70} \mathrm{Ge}$ is again considered. As before in Sec. 2.1.1, since $Q=S_{p}\left({ }^{71} \mathrm{Ge}\right)-S_{n}\left({ }^{71} \mathrm{Ge}\right)>0$ the reaction rates can be 
found in the regular rate file. Table II shows a small part of that table. Looking for ${ }^{70} \mathrm{Ga}$, for instance by a text search for "ga70", one first encounters the data block for ${ }^{70} \mathrm{Ga}+\mathrm{n}$. Farther down one finds the data block ${ }^{70} \mathrm{Ga}+\mathrm{p}$ since for the same target isotope the data blocks are sorted by reaction. With a computer code it may be more convenient to search for the numbers for charge, mass number and projectile at the beginning of the line. The relevant identification line tells us that the values for the $(\mathrm{p}, \mathrm{n})$ as well as the $(\mathrm{p}, \alpha)$ reaction are printed. Unlike the cross section table, which starts with $L$ projectile energy values, the temperature grid is not given here because the rates are always calculated at the same 24 stellar temperatures given above. The first line is immediately followed by the rates for the capture reaction, first 24 values of laboratory rates, then 24 stellar rates. These are followed by pairs of 24 laboratory and stellar rates each for the $(\mathrm{p}, \mathrm{n})$ and the $(\mathrm{p}, \alpha)$ reactions. Thus, for instance, the value of the ${ }^{70} \mathrm{Ga}(\mathrm{p}, \mathrm{n}){ }^{70} \mathrm{Ge}$ laboratory rate at $T_{9}=9$ (next to last position in the third line) is given as $7.27 \times 10^{6} \mathrm{~cm}^{3} \mathrm{~s}^{-1} \mathrm{~mol}^{-1}$. This should be compared to the stellar rate of $6.94 \times 10^{6} \mathrm{~cm}^{3} \mathrm{~s}^{-1} \mathrm{~mol}^{-1}$. The RATH tables should always be consulted to determine if the statistical model is suited for predicting cross sections for a specific reaction at a given rate (see Sec. 2.5.1).

The values for the inverse reaction ${ }^{70} \mathrm{Ge}(\mathrm{n}, \mathrm{p}){ }^{70} \mathrm{Ga}$ cannot be extracted from the file as $Q<0$ for this reaction. An inspection of the identification line for ${ }^{70} \mathrm{Ge}+\mathrm{n}$ further down shows that $b=0$ and that therefore no rates are printed for this reaction. Reverse stellar rates for particle reactions can easily be computed as explained in the following section.

The above instructions are relevant to the posted rate files rates_frdm.asc and rates_etfsiq.asc.

\subsection{Reverse Stellar Rates and Endothermic Laboratory Cross Sections}

The data presented in Secs. 2.1 and 2.2 correspond to the RATH tables. Since reverse astrophysical rates can easily be calculated by detailed balance, the previous RATH work only gave such rates explicitly for exothermic reactions and capture reactions and provided the means to derive the fit parameters for the reverse rates. The reverse stellar rate is also not explicitly given here but can easily be derived for each temperature with the following formulas. For reactions with particles in all channels use

$$
N_{A}\langle\sigma v\rangle_{\mathrm{rev}}^{*}=N_{A}\langle\sigma v\rangle^{*} \frac{G_{i}(T)}{G_{m}(T)} e^{f}
$$

In order to compute a photodisintegration rate $\lambda_{\gamma}^{*}\left(\right.$ in $\left.\mathrm{s}^{-1}\right)$ from a capture rate use

$$
\lambda_{\gamma}^{*}=\frac{G_{i}(T)}{G_{m}(T)} T_{9}^{3 / 2} N_{A}\langle\sigma v\rangle^{*} e^{f}
$$


The exponent $f$ is defined as

$$
f=a_{0}^{\mathrm{rev}}-a_{0}-\frac{11.6045 Q}{T_{9}} .
$$

The quantities $G_{i}, G_{m}$ are the partition functions of the target and residual nucleus, respectively, in the forward reaction, i.e. the reaction $x+i \rightarrow m+y$, where $x$ can be a particle and $y$ can be a particle or a $\gamma$-ray (compare Eqs. $12-$ 14 in $\mathrm{RATH}$ ). The parameters $a_{0}, a_{0}^{\text {rev }}$ and the reaction $Q$ value (in $\mathrm{MeV}$ ) for the forward reaction as well as the required partition functions can be looked up in the RATH tables. The $Q$ value can also be calculated from the particle separation energies given with the cross sections.

It has to be emphasized that detailed balance can only be applied to stellar rates and not to laboratory rates or cross sections with the target being in the ground state. When comparing to experiments it can sometimes be useful to also have access to cross sections for reactions with negative $Q$ values. For that purpose, calculated cross sections for such endothermic reactions are given in two additional files: rates_frdm_endo.asc and rates_etfsiq_endo.asc (one file for each mass model). The format is the same as for the cross sections discussed in Sec. 2.1. Here, only the energies are given for which the cross sections are not zero, i.e. above the particle thresholds. Cross sections at lower energies can be assumed to be zero.

Photodisintegration cross sections for targets in the ground state are not given here. They are discussed elsewhere [4, 5] and a table will be published separately $[6]$.

\subsection{Experimental Nuclear Level Schemes}

The experimental level information utilized in the statistical model calculations is provided in the last file. Up to 20 experimental levels (including the ground state) were considered. The states are taken from [7], up to the first level for which the spin or parity assignment was not known. Above this cut-off a theoretical nuclear level density $[\mathbb{\&}$ was employed. Ground state spin and parities are known for many unstable nuclei. Far off stability, where experimental values are not known, ground state spins and parities are derived from the neutron and proton single-particle spins given in [- by applying Nordheim rules. However, tabulated here are only the experimental spin and parity values used because the complete list of ground state spins is already given in RATH. This information is important when comparing with other statistical model calculations.

\subsubsection{Instructions for the Use of the Nuclear Level File}

An example is shown in Table III. The isotopes are ordered first by charge and then by mass number. The data block for each isotope starts with an identification line specifying, in this order: charge and mass number of the isotope, number $L$ of experimental nuclear levels given, and finally the isotope

again written in element notation. The succeeding lines specify the $L$ nuclear 
levels, giving for each level the excitation energy (in $\mathrm{MeV}$ ) as well as the parity and the spin assignment. The parity is coded as a sign before the spin. There are at most five levels per line. A new isotope entry again starts with an identification line.

As an example, consider the nucleus ${ }^{70} \mathrm{Ga}$ in Table III, which in turn shows a small fraction of the full file. It can be seen that five experimental levels were used in the calculation, including the ground state. The list starts with the $J^{\pi}=1^{+}$ground state, while the third excited state of ${ }^{70} \mathrm{Ga}$ is located at an energy of $0.6909 \mathrm{MeV}$ and has a spin and parity assignment of $J^{\pi}=2^{-}$.

The above instructions are relevant to the posted file levels.asc.

\subsection{Caveats}

The cross sections and reaction rates provided here should only be used keeping in mind the fundamental limitations of the model as discussed below.

\subsubsection{Applicability of the Statistical Model}

The statistical model can be applied provided that the use of averaged transmission coefficients (Eq. 3 in RATH) is permitted. This will be the case for high level densities with completely overlapping resonances, typical for the compound nucleus reaction mechanism. For light nuclei, decreasing particle separation energies, or at shell closures, level densities will eventually become too low for the application of the statistical model at astrophysical temperatures. In those cases, single resonances and contributions from the direct reaction mechanism have to be taken into account $[10]$. Based on a level density description, a quantitative criterion for the applicability was derived recently [8]. The lower temperature limit of the validity of the statistical model for the calculation of reaction rates is already given in the RATH tables. It is advisable to check those limits before using the cross sections and rates given in this paper.

In principle, a similar criterion can also be derived for cross sections when comparing to experimental data. In the case of a nuclear experiment, the energy distribution over which one has to average is not determined by a MaxwellBoltzmann velocity distribution but by the uncertainty in the beam energy and the energy straggling in the target. Since these differ depending on the experimental setup it is not possible to give a global criterion. However, as a first approximation one can use the temperature limits given in RATH.

\subsubsection{Validity of the Predictions}

It should be noted that only purely theoretical rates are given here which do not use any direct experimental information (except for nuclear masses and ground and excited state information where available). The methods to predict nuclear properties needed in the statistical model calculations are chosen to be as reliable as possible in order to retain predictive power. Nevertheless, the uncertainties and some known weaknesses (e.g. of the FRDM around shell closures) of the 
input will be reflected in the predicted values. This is a compromise which may lead to locally enhanced inaccuracies but it emphasizes the importance of reliable predictions of rates far off stability (see RATH). If one would use experimental data (such as level densities) and locally tuned parametrizations of nuclear properties (optical potentials, Giant Dipole Resonance widths) as input for our statistical model calculation, a further increase in accuracy could be achieved. Since the main scope of this work is the prediction of nuclear reaction rates for astrophysics for experimentally unaccessible nuclei, a global approach is better suited. Therefore, in comparison to experimental work deviations within a factor of $1.5-2$ could appear although the average deviation will be smaller. For neutron capture it has been shown that the average deviation is about a factor of $1.3-1.4[8]$.

\section{Summary}

Nuclear cross sections and thermonuclear reaction rates for neutron-, protonand $\alpha$-induced reactions and their inverses have been calculated in the statistical model and published in the previous paper RATH [1]. All cross sections and rates from $\mathrm{Ne}$ to $\mathrm{Bi}$ are given from proton dripline to neutron dripline, thus covering a considerable part of the nuclear chart. Fits to reaction rates have been provided in RATH for two sets of rates, calculated with input from two different mass models. Here, we make accessible the input data for the original calculation, containing nuclear cross sections as well as reaction rates. Excitation energy, spin and parity are quoted for the experimental level information used in the calculations to facilitate comparison with other models. Further details on the underlying models are given in RATH.

In real applications, these rates should be supplemented or replaced by experimental rates as they become available. Such a combination of theoretical and experimental rates is provided, e.g., in the REACLIB compilation. Further details on REACLIB, the NON-SMOKER code, and the calculations are presented

at http: // quasar. physik. unibas.ch/ 'tommy/reaclib.html. Rates based on additional mass models can also be obtained from the authors on request or directly at the latter URL.

\section{Acknowledgements}

This work was supported in part by the Swiss National Science Foundation (grant 2000-053798.98) and the Austrian Academy of Sciences (APART). T. R. acknowledges a PROFIL professorship of the Swiss National Science Foundation (grant 2124-055832.98).

\section{References}

[1] T. Rauscher and F.-K. Thielemann, Atomic Data Nucl. Data Tabl. 75, 1 (2000), doi:10.1006/adnd.2000.0834. 
[2] P. Möller, J. R. Nix, W. D. Myers, and W. J. Swiatecki, Atomic Data Nucl. Data Tabl. 59, 185 (1995), doi:10.1006/adnd.1995.1002.

[3] J. M. Pearson, R. C. Nayak, and S. Goriely, Phys. Lett. B 387, 455 (1996).

[4] P. Mohr et al., Phys. Lett. B 488, 127 (2000).

[5] K. Vogt et al., Phys. Rev. C, submitted (2001).

[6] T. Rauscher, in preparation (unpublished).

[7] R. B. Firestone, in Table of Isotopes, 8th ed., edited by V. S. Shirley (Wiley, New York, 1996).

[8] T. Rauscher, F.-K. Thielemann, and K.-L. Kratz, Phys. Rev. C 56, 1613 (1997).

[9] P. Möller, J. R. Nix, and K.-L. Kratz, Atomic Data Nucl. Data Tabl. 66, 131 (1997), doi:10.1006/adnd.1997.0746.

[10] T. Rauscher et al., Phys. Rev. C 57, 2031 (1998). 


\section{Explanation of Tables}

\section{Table I: Sample of Cross Section Tables}

Sample excerpt of the ASCII file $c s \_f r d m$.asc containing cross sections for reactions on the ground state calculated using input data from the FRDM mass model. See RATH for details.

Z Charge number of target

A Mass number of target

$\mathrm{p} \quad$ Projectile: 0 for neutron, 1 for proton, 2 for $\alpha$ particle

L Number of calculated energies and cross sections

b Index $b$, which assumes values of 0 or 1 depending on whether cross sections are listed $(b=1)$ for the first particle exit channel (defined by column $\mathrm{f}$ )

c Index $c$, which assumes values of 0 or 1 depending on whether cross sections are listed $(c=1)$ for the second particle exit channel (defined by column $\mathrm{h}$ )

Zc Charge number of the compound nucleus

Ac Mass number of the compound nucleus

SN Neutron separation energy in the compound nucleus, in $\mathrm{MeV}$

SP Proton separation energy in the compound nucleus, in $\mathrm{MeV}$

SA $\quad \alpha$-particle separation energy in the compound nucleus, in $\mathrm{MeV}$

$\mathrm{T} \quad$ Target written as combination of element name and mass number

$\mathrm{x} \quad$ Projectile: $+\mathrm{n}$ for neutron, $+\mathrm{p}$ for proton, $+\mathrm{a}$ for $\alpha$ particle

$\mathrm{e}, \mathrm{f}, \mathrm{h} \quad$ Exit channels: $-\mathrm{g}$ for $\gamma$ ray (capture reaction), $-\mathrm{n}$ for neutron, $-\mathrm{p}$ for proton, $-\mathrm{a}$ for $\alpha$ particle. In this table, cross sections for the capture channel are always listed and column e is always $-\mathrm{g}$; in the table of reactions with $Q<0$ (the $c s_{-} f r d m_{-}$endo.asc and cs_etfsiq_endo.asc files), no capture reactions are given and column $\mathrm{e}$ is omitted.

E1...EL Center-of-mass energies in $\mathrm{MeV}$ for which the nuclear reaction cross sections have been calculated. There are $L$ values with up to eight entries per line. The energies are only given once as they are the same for the same combination of target and projectile.

G1...GL Capture cross sections in barn; $L$ values are given with up to eight entries per line. Values lower than $10^{-30}$ barn are set equal to zero. 
(X1...XL) Nuclear cross sections for the first particle exit channel (specified by column $\mathrm{f}$ ) in barn. $L$ values are given with up to eight entries per line. They are listed only if $b=1$. Values lower than $10^{-30}$ barn are set equal to zero.

(Y1... YL) Nuclear cross sections for the second particle channel (specified by column h) in barn; $L$ values are given with up to eight entries per line. They are listed only if $c=1$. Values lower than $10^{-30}$ barn are set equal to zero.

\section{Table II: Sample of Rate Tables}

Sample of the ASCII file rates frdm.asc of laboratory rates $N_{A}\langle\sigma v\rangle^{\text {lab }}$ calculated with the ground state cross sections of Table I and stellar rates $N_{A}\langle\sigma v\rangle^{*}$ calculated from stellar cross sections with a thermally excited target, given in $\mathrm{cm}^{3} \mathrm{~s}^{-1} \mathrm{~mol}^{-1}$. Values lower than $10^{-30} \mathrm{~cm}^{3} \mathrm{~s}^{-1} \mathrm{~mol}^{-1}$ are set equal to zero.

Z Charge number of target

A Mass number of target

$\mathrm{p} \quad$ Projectile: 0 for neutron, 1 for proton, 2 for $\alpha$ particle

b Index $b$, which assumes values of 0 or 1 depending on whether rates are listed $(b=1)$ for the first particle exit channel (defined by column f)

c Index $c$, which assumes values of 0 or 1 depending on whether rates are listed $(c=1)$ for the second particle exit channel (defined by column h)

$\mathrm{T} \quad$ Target written as combination of element name and mass number

$\mathrm{x} \quad$ Projectile: $+\mathrm{n}$ for neutron, $+\mathrm{p}$ for proton, $+\mathrm{a}$ for $\alpha$ particle

$\mathrm{e}, \mathrm{f}, \mathrm{h} \quad$ Exit channels: $-\mathrm{g}$ for $\gamma$ ray (capture reaction), $-\mathrm{n}$ for neutron, $-\mathrm{p}$ for proton, $-\mathrm{a}$ for $\alpha$ particle. Rates for the capture channel are always listed and column e is always $-\mathrm{g}$.

G1...G24 Laboratory capture rates $N_{A}\langle\sigma v\rangle^{\text {lab }}$ (see Sec. 2.2). Eight entries each in three lines are given, corresponding to the grid of 24 temperatures $T_{9}=0.1,0.15,0.2,0.3,0.4,0.5,0.6,0.7,0.8,0.9,1.0,1.5$, $2.0,2.5,3.0,3.5,4.0,4.5,5.0,6.0,7.0,8.0,9.0,10.0\left(T_{9}\right.$ is given in $\left.10^{9} \mathrm{~K}\right)$.

H1... H24 Stellar capture rates $N_{A}\langle\sigma v\rangle^{*}$ (see Eq. 10 in RATH), given as eight entries each in three lines for the 24 temperature grid. Only the stellar rate should be used in astrophysical applications and when applying detailed balance in order to derive the reverse rate. 
(R1...R24) Laboratory rates for the first particle exit channel (specified by column $\mathrm{f}$ ) at 24 temperatures. They are listed only if $b=1$.

(U1... U24) Stellar rates for the first particle channel (specified by column f). They are listed only if $b=1$. Only the stellar rate should be used in astrophysical applications and when applying detailed balance in order to derive the reverse rate.

(R1...R24) Laboratory rates for the second particle exit channel (specified by column h) at 24 temperatures. They are listed only if $c=1$.

(U1... U24) Stellar rates for the second particle channel (specified by column h). They are listed only if $c=1$. Only the stellar rate should be used in astrophysical applications and when applying detailed balance in order to derive the reverse rate.

\section{Table III: Sample of Nuclear Level Table}

Up to 20 experimentally known nuclear states were used in the calculation (see Sec. 2.4). An excerpt of the full on-line ASCII file levels.asc is given here as an example. Only the experimental values used in the calculation are listed. The theoretical ground state spins were already given in RATH.

Z Charge number of the isotope

A Mass number of the isotope

L Number of given energy levels (including the ground state)

$\mathrm{T} \quad$ Isotope written as a combination of element name and mass number

E1...EL Excitation energy of the level, given in $\mathrm{MeV}$

p1...pL Parity of the level

$\mathrm{J} 1 \ldots \mathrm{JL} \quad$ Spin of the level 


\section{Table I: Sample of Cross Section Tables}

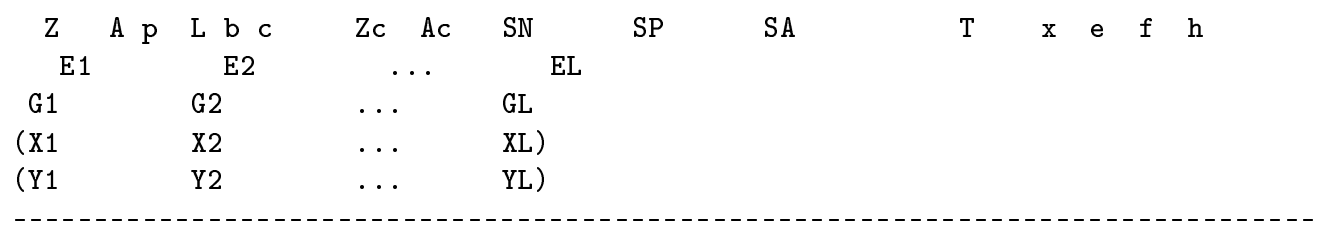

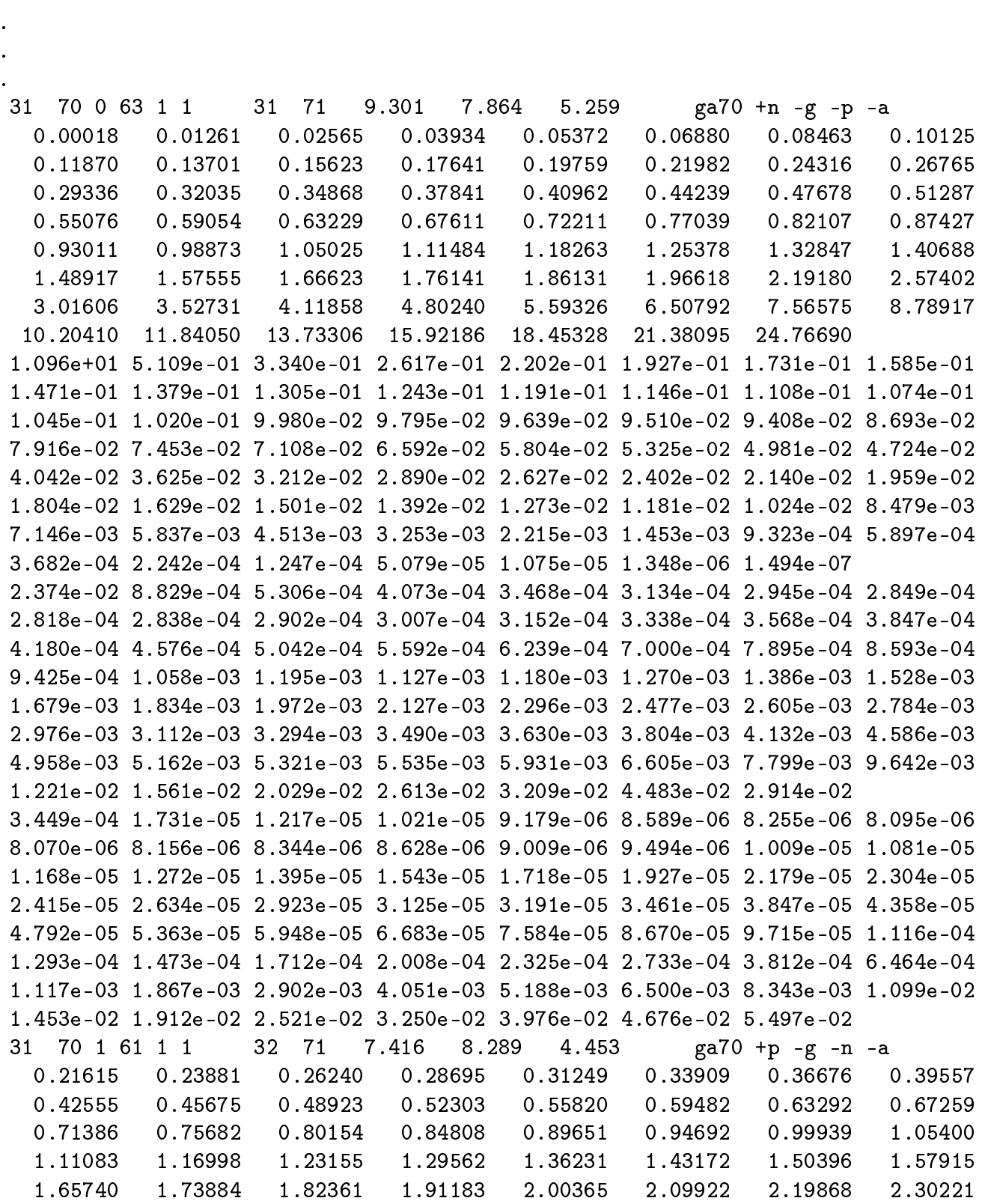




$\begin{array}{rrrrrrrr}2.40995 & 2.52208 & 2.88669 & 3.29775 & 3.76119 & 4.28367 & 4.87273 & 5.53684 \\ 6.28557 & 7.12969 & 8.08137 & 9.15430 & 10.36395 & 11.72771 & 13.26524 & 14.99867 \\ 16.95297 & 19.15627 & 21.64030 & 24.44082 & 25.45150 & & & \end{array}$

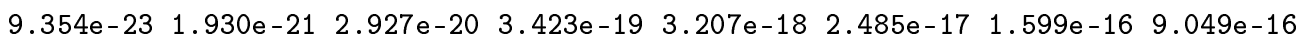
$\begin{array}{llllllll}4.526 e-15 & 2.027 e-14 & 8.233 e-14 & 3.064 e-13 & 1.054 e-12 & 3.380 e-12 & 1.017 e-11 & 2.889 e-11\end{array}$ $\begin{array}{llllllll}7.788 e-11 & 2.002 e-10 & 4.925 e-10 & 1.090 e-09 & 2.363 e-09 & 5.056 e-09 & 1.052 e-08 & 2.128 e-08\end{array}$ 4.191e-08 8.056e-08 $1.514 \mathrm{e}-07 \quad 2.487 \mathrm{e}-07 \quad 4.151 \mathrm{e}-07 \quad 7.153 \mathrm{e}-07 \quad 1.195 \mathrm{e}-06 \quad 1.989 \mathrm{e}-06$ $3.186 e-06 \quad 4.893 e-06 \quad 7.600 e-06 \quad 1.171 e-05 \quad 1.771 e-05 \quad 2.577 e-05 \quad 3.738 e-05 \quad 5.347 e-05$

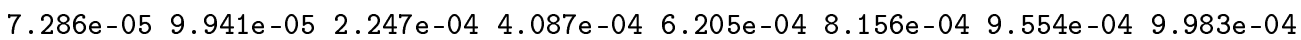
$\begin{array}{lllllllll}9.314 e-04 & 7.851 e-04 & 6.312 e-04 & 5.364 e-04 & 4.253 e-04 & 2.974 e-04 & 1.839 e-04 & 9.549 e-05\end{array}$ $4.496 \mathrm{e}-051.745 \mathrm{e}-05 \quad 5.796 \mathrm{e}-06 \quad 1.387 \mathrm{e}-06 \quad 7.919 \mathrm{e}-07$

$\begin{array}{llllllll}6.777 e-22 & 1.498 e-20 & 2.418 e-19 & 3.001 e-18 & 2.981 e-17 & 2.447 e-16 & 1.707 e-15 & 1.029 e-14\end{array}$ $5.469 e-14 \quad 2.597 e-13 \quad 1.116 e-12 \quad 4.386 e-12 \quad 1.590 e-11 \quad 5.359 e-11 \quad 1.690 e-10 \quad 5.018 e-10$ $\begin{array}{llllllll}1.409 e-09 & 3.761 e-09 & 9.575 e-09 & 2.341 e-08 & 5.491 e-08 & 1.239 e-07 & 2.698 e-07 & 5.685 e-07\end{array}$ $\begin{array}{llllllll}1.161 e-06 & 2.302 e-06 & 4.441 e-06 & 8.373 e-06 & 1.537 e-05 & 2.752 e-05 & 4.817 e-05 & 8.244 e-05\end{array}$ $\begin{array}{llllllll}1.382 e-04 & 2.271 e-04 & 3.656 e-04 & 5.771 e-04 & 8.935 e-04 & 1.358 e-03 & 2.025 e-03 & 2.964 e-03\end{array}$ $4.260 \mathrm{e}-03 \quad 6.005 \mathrm{e}-03 \quad 1.503 \mathrm{e}-02 \quad 3.191 \mathrm{e}-02 \quad 5.837 \mathrm{e}-02 \quad 9.500 \mathrm{e}-02 \quad 1.421 \mathrm{e}-01 \quad 1.973 \mathrm{e}-01$ $2.569 e-013.147 e-01 \quad 3.793 e-01 \quad 4.956 e-01 \quad 6.171 e-01 \quad 6.951 e-01 \quad 7.324 e-01 \quad 7.529 e-01$ $6.897 \mathrm{e}-015.214 \mathrm{e}-01 \quad 3.469 \mathrm{e}-01 \quad 1.634 \mathrm{e}-01 \quad 1.151 \mathrm{e}-01$

$2.036 e-27 \quad 4.709 e-26 \quad 8.070 e-25 \quad 1.073 e-23 \quad 1.150 e-22 \quad 1.025 e-21 \quad 7.597 e-21 \quad 4.990 e-20$ $2.911 e-19 \quad 1.528 e-18 \quad 7.305 e-18 \quad 3.215 e-17 \quad 1.314 e-16 \quad 5.026 e-16 \quad 1.812 e-15 \quad 6.195 e-15$ $\begin{array}{llllllll}2.017 e-14 & 6.290 e-14 & 1.885 e-13 & 5.038 e-13 & 1.330 e-12 & 3.499 e-12 & 9.008 e-12 & 2.266 e-11\end{array}$ $\begin{array}{lllllllll}5.576 e-11 & 1.344 e-10 & 3.180 e-10 & 6.749 e-10 & 1.444 e-09 & 3.170 e-09 & 6.731 e-09 & 1.434 e-08\end{array}$ $\begin{array}{llllllll}2.971 e-08 & 5.848 e-08 & 1.169 e-07 & 2.321 e-07 & 4.542 e-07 & 8.506 e-07 & 1.594 e-06 & 2.944 e-06\end{array}$ 5.170e-06 9.104e-06 $4.393 e-05 \quad 1.681 e-04 \quad 5.223 e-04 \quad 1.348 e-03 \quad 2.921 e-03 \quad 5.263 e-03$

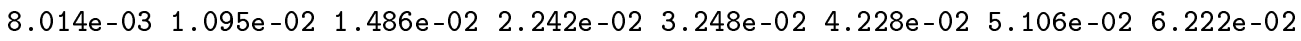
$\begin{array}{llll}1.378 e-01 & 2.611 e-012.296 e-01 & 1.002 e-01 & 6.595 e-02\end{array}$

\begin{tabular}{|c|c|c|c|c|c|c|c|}
\hline $\begin{array}{llll}1 & 70 & 2 & 65\end{array}$ & 500 & $33 \quad 74$ & 7.975 & 4.379 & ga70 & $+a-g-n$ & $-p$ \\
\hline 0.64179 & 0.64903 & 0.65743 & 0.66717 & 0.67845 & 0.69153 & 0.70668 & 0.72425 \\
\hline .74461 & 0.76821 & 0.79557 & 0.82727 & 0.86402 & 0.90662 & 0.95598 & 1.01320 \\
\hline 1.07952 & 1.15639 & 1.24548 & 1.34875 & 1.46844 & 1.60716 & 1.76796 & 1.95432 \\
\hline 2.17033 & 2.42069 & 2.71087 & 3.48925 & 3.55116 & 3.59539 & 3.61420 & 3.63436 \\
\hline 3.65595 & 3.67908 & 3.70385 & 3.73038 & 3.75881 & 3.78925 & 3.82186 & 3.85679 \\
\hline 3.89421 & 3.93429 & 3.97722 & 4.02320 & 4.07246 & 4.12522 & 4.18174 & 4. 24227 \\
\hline 4.4 & 4.70747 & 5.02270 & 5.41011 & 5.88625 & 6.47142 & 7.19060 & 8.07447 \\
\hline 9.16075 & 0.49579 & 2.13655 & 14.15305 & 16.63133 & 19.67714 & 23.42044 & 28.02096 \\
\hline
\end{tabular}

29.77768

$\begin{array}{llllllllllll}0.000 e+00 & 0.000 e+00 & 0.000 e+00 & 0.000 e+00 & 0.000 e+00 & 0.000 e+00 & 0.000 e+00 & 0.000 e+00\end{array}$ $\begin{array}{lllllllllll}0.000 e+00 & 0.000 e+00 & 0.000 e+00 & 0.000 e+00 & 0.000 e+00 & 1.084 e-35 & 2.645 e-34 & 7.954 e-33\end{array}$ $\begin{array}{llllllll}2.899 e-31 & 1.255 e-29 & 6.292 e-28 & 3.551 e-26 & 2.184 e-24 & 1.417 e-22 & 9.370 e-21 & 6.095 e-19\end{array}$

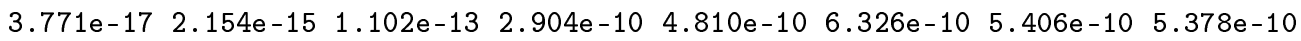
$\begin{array}{llllllll}5.603 e-10 & 5.073 e-10 & 4.458 e-10 & 4.625 e-10 & 5.058 e-10 & 5.732 e-10 & 6.667 e-10 & 7.851 e-10\end{array}$ $9.441 \mathrm{e}-101.160 \mathrm{e}-09 \quad 1.453 \mathrm{e}-09 \quad 1.734 \mathrm{e}-09 \quad 1.611 \mathrm{e}-09 \quad 1.783 \mathrm{e}-09 \quad 2.058 \mathrm{e}-09 \quad 2.492 \mathrm{e}-09$ 5.025e-09 $1.269 e-08 \quad 4.171 e-08 \quad 1.707 e-07 \quad 7.990 e-07 \quad 3.932 e-06 \quad 1.861 e-05 \quad 7.506 e-05$ $2.181 e-04 \quad 3.652 e-04 \quad 3.597 e-04 \quad 2.483 e-04 \quad 1.177 e-04 \quad 2.259 e-05 \quad 1.377 e-06 \quad 4.875 e-08$ $1.987 e-08$

\begin{tabular}{|c|c|c|c|c|c|c|c|}
\hline $\begin{array}{llll}71 & 0 & 60\end{array}$ & 01 & 31 & 6.521 & 5.467 & ga71 & $+n-g-p$ & $-a$ \\
\hline 0.00018 & 0.00423 & 0.00900 & 0.01463 & 0.02125 & 0.02907 & 0.03828 & 0.04914 \\
\hline 0.06193 & 0.07702 & 0.09480 & 0.11576 & 0.14047 & 0.16960 & 0.20393 & 0.24440 \\
\hline 0.29211 & 0.34834 & 0.41463 & 0.49277 & 0.58487 & 0.69345 & 0.82143 & 0.97229 \\
\hline 1.15011 & 1.35973 & 1.60682 & 1.89809 & 2.24142 & 2.27455 & 2.30962 & 2.34677 \\
\hline 2.38610 & 2.42775 & 2.47186 & 2.51857 & 2.56803 & 2.62041 & 2.67588 & 2.73461 \\
\hline 2.79681 & 2.86268 & 3.08450 & 3.34790 & 3.66070 & 4.03213 & 4.47320 & 4.99696 \\
\hline 5.61892 & 6.35749 & 7.23452 & 8.27598 & 9.51269 & 10.98127 & 12.72517 & 14.79603 \\
\hline
\end{tabular}


$4.126 e+003.253 e-01 \quad 1.957 e-01 \quad 1.436 e-01 \quad 1.135 e-01 \quad 9.312 e-02 \quad 7.829 e-02 \quad 6.698 e-02$ 5.811e-02 5.102e-02 $4.524 e-02 \quad 4.048 e-02 \quad 3.651 e-02 \quad 3.321 e-02 \quad 3.045 e-02 \quad 2.817 e-02$ $2.632 \mathrm{e}-02 \quad 2.486 \mathrm{e}-02 \quad 2.316 \mathrm{e}-02 \quad 1.990 \mathrm{e}-02 \quad 1.483 \mathrm{e}-02 \quad 1.243 \mathrm{e}-02 \quad 1.120 \mathrm{e}-02 \quad 1.033 \mathrm{e}-02$ $8.538 \mathrm{e}-03 \quad 7.404 \mathrm{e}-03 \quad 5.946 \mathrm{e}-03 \quad 5.439 \mathrm{e}-03 \quad 5.251 \mathrm{e}-03 \quad 5.225 \mathrm{e}-03 \quad 5.169 \mathrm{e}-03 \quad 5.132 \mathrm{e}-03$ 5.093e-03 5.032e-03 $4.976 e-03 \quad 4.923 e-03 \quad 4.871 e-03 \quad 4.783 e-03 \quad 4.714 e-03 \quad 4.647 e-03$ $4.552 e-03 \quad 4.461 e-03 \quad 4.157 e-03 \quad 3.789 e-03 \quad 3.351 e-03 \quad 2.848 e-03 \quad 2.310 e-03 \quad 1.757 e-03$ $1.264 e-03 \quad 8.680 e-04 \quad 5.741 e-04 \quad 3.699 e-04 \quad 3.043 e-04 \quad 3.016 e-04 \quad 4.249 e-04 \quad 5.691 e-04$ $2.808 e-04 \quad 3.937 e-05 \quad 3.016 e-06 \quad 1.434 e-07$

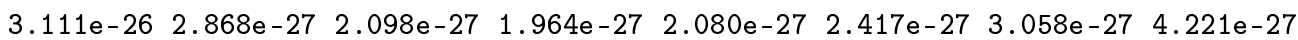
$6.396 e-27 \quad 1.073 e-26 \quad 2.016 e-26 \quad 4.292 e-26 \quad 1.049 e-25 \quad 2.980 e-25 \quad 9.974 e-25 \quad 3.981 e-24$ $1.915 e-23 \quad 1.119 e-22 \quad 7.479 e-22 \quad 5.784 e-21 \quad 4.563 e-20 \quad 4.925 e-19 \quad 6.664 e-18 \quad 1.025 e-16$



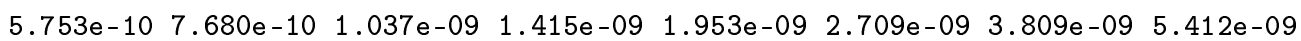
7.733e-09 $1.117 \mathrm{e}-08 \quad 3.540 \mathrm{e}-08 \quad 1.195 \mathrm{e}-07 \quad 4.216 \mathrm{e}-07 \quad 1.517 \mathrm{e}-06 \quad 5.442 \mathrm{e}-06 \quad 1.875 \mathrm{e}-05$ 6.141e-05 $1.874 \mathrm{e}-04 \quad 5.147 \mathrm{e}-04 \quad 1.214 \mathrm{e}-03 \quad 3.119 \mathrm{e}-03 \quad 8.642 \mathrm{e}-03 \quad 3.286 \mathrm{e}-02 \quad 1.408 \mathrm{e}-01$ $4.250 \mathrm{e}-016.735 \mathrm{e}-01 \quad 7.452 \mathrm{e}-01 \quad 9.032 \mathrm{e}-01$

(3)

\begin{tabular}{|c|c|c|c|c|c|c|c|}
\hline 550 & 11 & 56 & -1 & 4 & $g$ & $+n$ & \\
\hline 0.00018 & 0.00662 & 0.01337 & 0.02046 & 0.02789 & 0.03569 & 0.04387 & 0.05245 \\
\hline 0.06145 & 0.07090 & 0.08081 & 0.09121 & 0.10211 & 0.11356 & 0.12557 & 0.13816 \\
\hline 0.15138 & 0.16524 & 0.17979 & 0.19505 & 0.21106 & 0.22786 & 0.24549 & 0.26398 \\
\hline 0.28337 & 0.30373 & 0.32508 & 0.34748 & 0.37098 & 0.39564 & 0.42150 & 0.44864 \\
\hline 0.47712 & 0.50699 & 0.53833 & 0.57121 & 0.60570 & 0.64189 & 0.67986 & 0.71970 \\
\hline 0.76149 & 0.80534 & 0.85134 & 0.89960 & 0.95023 & 1.11755 & 1.31077 & 1.53390 \\
\hline 1.79156 & 2.08911 & 2.43271 & 2.82949 & 3.28769 & 3.81681 & 4.42782 & 5.13341 \\
\hline 5.94822 & 6.88914 & 7.97569 & 9.23043 & 10.67938 & 12.35259 & 12.96602 & \\
\hline
\end{tabular}

$\begin{array}{llllllll}6.622 e-04 & 1.076 e-04 & 7.536 e-05 & 6.094 e-05 & 5.237 e-05 & 4.655 e-05 & 4.229 e-05 & 3.904 e-05\end{array}$ $\begin{array}{llllllll}3.644 e-05 & 3.431 e-05 & 3.255 e-05 & 3.106 e-05 & 2.978 e-05 & 2.869 e-05 & 2.774 e-05 & 2.692 e-05\end{array}$

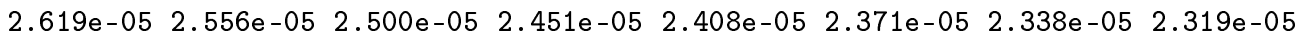
$2.294 e-05 \quad 2.273 e-05 \quad 2.254 e-05 \quad 2.238 e-05 \quad 2.225 e-05 \quad 2.253 e-05 \quad 2.243 e-05 \quad 2.235 e-05$

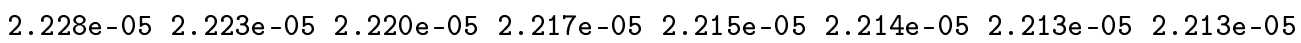
$2.213 e-05 \quad 2.212 e-05 \quad 2.211 e-05 \quad 2.237 e-05 \quad 2.234 e-05 \quad 2.217 e-05 \quad 2.188 e-05 \quad 2.152 e-05$

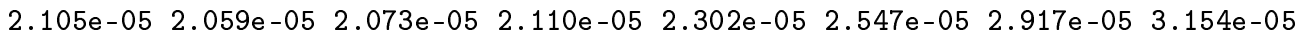
$3.022 \mathrm{e}-05 \quad 2.724 \mathrm{e}-05 \quad 2.548 \mathrm{e}-05 \quad 2.534 \mathrm{e}-05 \quad 2.481 \mathrm{e}-05 \quad 2.285 \mathrm{e}-05 \quad 2.177 \mathrm{e}-05$

$\begin{array}{lllllllll}6.033 e+01 & 9.802 e+00 & 6.859 e+00 & 5.544 e+00 & 4.761 e+00 & 4.229 e+00 & 3.840 e+00 & 3.540 e+00\end{array}$

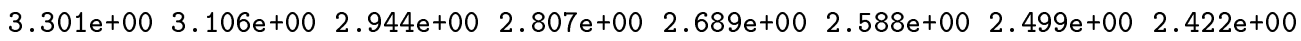
$\begin{array}{llllllll}2.354 e+00 & 2.294 e+00 & 2.241 e+00 & 2.194 e+00 & 2.152 e+00 & 2.115 e+00 & 2.081 e+00 & 2.061 e+00\end{array}$ $2.035 \mathrm{e}+00 \quad 2.011 \mathrm{e}+00 \quad 1.990 \mathrm{e}+00 \quad 1.972 \mathrm{e}+00 \quad 1.955 \mathrm{e}+00 \quad 1.940 \mathrm{e}+00 \quad 1.927 \mathrm{e}+00 \quad 1.916 \mathrm{e}+00$

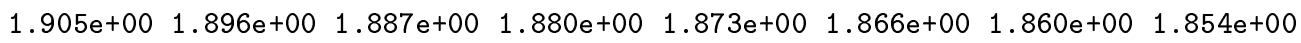

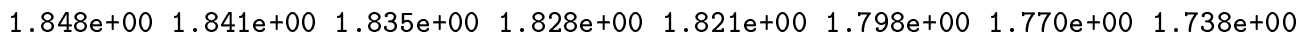
$\begin{array}{llllllll}1.705 e+00 & 1.678 e+00 & 1.661 e+00 & 1.660 e+00 & 1.676 e+00 & 1.699 e+00 & 1.714 e+00 & 1.708 e+00\end{array}$ $\begin{array}{lllllll}1.681 e+00 & 1.646 e+00 & 1.614 e+00 & 1.588 e+00 & 1.560 e+00 & 1.531 e+00 & 1.523 e+00\end{array}$

$\begin{array}{llllllll}3.348 e-02 & 5.449 e-03 & 3.819 e-03 & 3.093 e-03 & 2.661 e-03 & 2.369 e-03 & 2.156 e-03 & 1.993 e-03\end{array}$

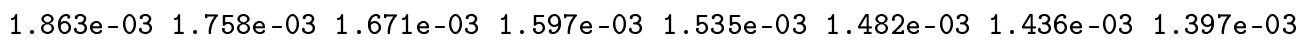
$1.362 e-03 \quad 1.333 e-03 \quad 1.308 e-03 \quad 1.286 e-03 \quad 1.267 e-03 \quad 1.251 e-03 \quad 1.238 e-03 \quad 1.232 e-03$ $1.223 e-03 \quad 1.216 e-03 \quad 1.211 e-03 \quad 1.208 e-03 \quad 1.206 e-03 \quad 1.226 e-03 \quad 1.226 e-03 \quad 1.228 e-03$

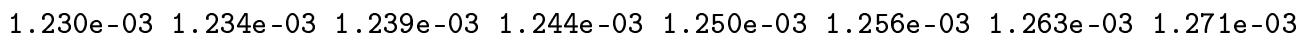

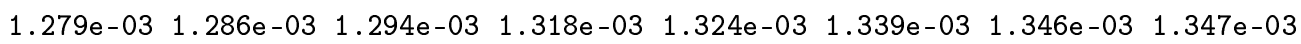
$\begin{array}{llllllll}1.340 e-03 & 1.337 e-03 & 1.386 e-03 & 1.478 e-03 & 1.722 e-03 & 2.050 e-03 & 2.496 e-03 & 2.836 e-03\end{array}$ $2.953 e-03 \quad 3.013 e-03 \quad 3.060 e-03 \quad 3.382 e-03 \quad 4.254 e-03 \quad 5.206 e-03 \quad 5.496 e-03$ 


\begin{tabular}{|c|c|c|c|c|c|c|c|}
\hline 700 & 01 & 32 & 16 & 4. & ge & $+n$ & $-a$ \\
\hline 0.00018 & 0.00385 & 0.00875 & 0.01528 & 0.02398 & 0.03559 & 0.05106 & 0.07170 \\
\hline 0.09923 & 0.13593 & 0.18488 & 0.25014 & 0.33718 & 0.45325 & 0.60803 & 0.81442 \\
\hline 1.08966 & 1.11732 & 1.14635 & 1.17683 & 1.20883 & 1. 24242 & 1.27769 & 1.31470 \\
\hline 1.35356 & 1.39436 & 1.43718 & 1.48214 & 1.52934 & 1.57888 & 1.63089 & 1.68549 \\
\hline 1.74281 & 1.80298 & 1.86614 & 1.93245 & 2.00207 & 2.07514 & 2.15185 & 2.23238 \\
\hline 2.31692 & 2.40567 & 2.49884 & 2.59664 & 2.69931 & 2.80710 & 2.92024 & 3.03902 \\
\hline 3.16371 & 3.29461 & 3.43203 & 3.88669 & 4.41268 & 5.02120 & 5.72518 & 6.53961 \\
\hline 7.48182 & 8.57184 & 9.83288 & 11.29176 & 12.97951 & 14.93206 & 17.19093 & 19.80419 \\
\hline
\end{tabular}

$22.82745 \quad 26.32501$

$3.742 e+003.301 e-01 \quad 2.001 e-01 \quad 1.466 e-01 \quad 1.156 e-01 \quad 9.436 e-02 \quad 7.872 e-02 \quad 6.697 e-02$ $\begin{array}{llllllll}5.820 e-02 & 5.162 e-02 & 4.679 e-02 & 4.362 e-02 & 4.206 e-02 & 4.200 e-02 & 4.349 e-02 & 4.716 e-02\end{array}$

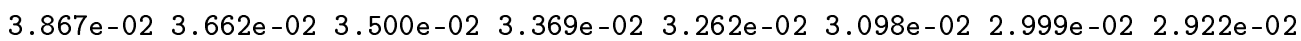

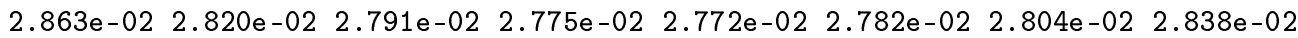
$\begin{array}{llllllll}2.712 e-02 & 2.651 e-02 & 2.622 e-02 & 2.617 e-02 & 2.634 e-02 & 2.671 e-02 & 2.729 e-02 & 2.541 e-02\end{array}$

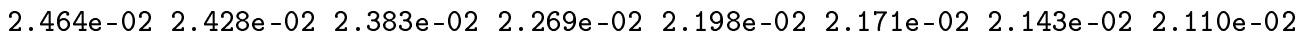

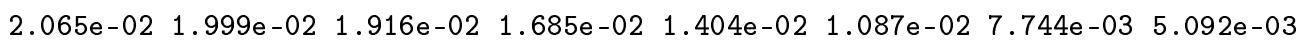
$\begin{array}{lllllllll}3.088 e-03 & 1.780 e-03 & 9.942 e-04 & 5.430 e-04 & 2.828 e-04 & 1.216 e-04 & 3.075 e-05 & 4.647 e-06\end{array}$ $5.384 \mathrm{e}-07 \quad 1.075 \mathrm{e}-07$

$2.169 \mathrm{e}-08$ 2.164e-09 $1.445 \mathrm{e}-09 \quad 1.160 \mathrm{e}-09 \quad 1.012 \mathrm{e}-09 \quad 9.343 \mathrm{e}-10 \quad 9.106 \mathrm{e}-10 \quad 9.480 \mathrm{e}-10$

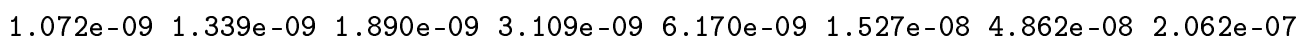

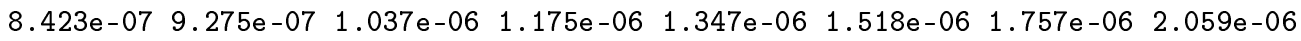
$2.440 \mathrm{e}-06 \quad 2.924 \mathrm{e}-06 \quad 3.541 \mathrm{e}-06 \quad 4.335 \mathrm{e}-06 \quad 5.363 \mathrm{e}-06 \quad 6.703 \mathrm{e}-06 \quad 8.465 \mathrm{e}-06 \quad 1.080 \mathrm{e}-05$ $1.302 \mathrm{e}-05 \quad 1.625 \mathrm{e}-05 \quad 2.066 \mathrm{e}-05 \quad 2.665 \mathrm{e}-05 \quad 3.485 \mathrm{e}-05 \quad 4.615 \mathrm{e}-05 \quad 6.188 \mathrm{e}-05 \quad 7.816 \mathrm{e}-05$

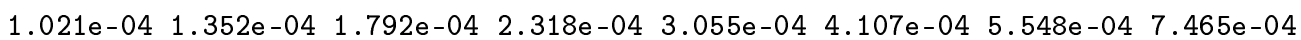

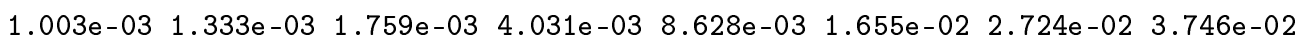
$\begin{array}{llllllll}4.420 e-02 & 5.027 e-02 & 5.884 e-02 & 7.042 e-02 & 8.494 e-02 & 1.133 e-01 & 1.958 e-01 & 1.222 e-01\end{array}$ $3.338 \mathrm{e}-02 \quad 1.103 \mathrm{e}-02$

\begin{tabular}{cccccccccc}
32 & 70179 & 0 & 1 & 33 & 71 & 11.623 & 4.621 & 3.440 & \multicolumn{2}{c}{ ge $70+\mathrm{p}-\mathrm{g}-\mathrm{n}$} & $-\mathrm{a}$ \\
0.22119 & 0.23140 & 0.24232 & 0.25398 & 0.26643 & 0.27974 & 0.29396 & 0.30915 \\
0.32538 & 0.34272 & 0.36125 & 0.38105 & 0.40219 & 0.42479 & 0.44893 & 0.47472 \\
0.50228 & 0.53172 & 0.56318 & 0.59678 & 0.63269 & 0.67105 & 0.71204 & 0.75583 \\
0.80261 & 0.85260 & 0.90601 & 0.96306 & 1.02402 & 1.08916 & 1.15874 & 1.23309 \\
1.31252 & 1.39739 & 1.48806 & 1.58493 & 1.68843 & 1.79901 & 1.91715 & 2.04338 \\
2.17824 & 2.32232 & 2.47626 & 2.64073 & 2.81645 & 3.00419 & 3.20478 & 3.41908 \\
3.64804 & 3.89267 & 4.15403 & 4.43327 & 4.73160 & 5.05035 & 5.39090 & 5.75474 \\
6.14348 & 6.55880 & 6.94929 & 6.98035 & 7.00253 & 7.03142 & 7.06414 & 7.10119 \\
7.14315 & 7.19068 & 7.24450 & 7.30546 & 7.37450 & 7.64153 & 8.02943 & 8.59292
\end{tabular}

$\begin{array}{llllllll}9.41147 & 10.60054 & 12.32785 & 14.83703 & 18.48201 & 23.77690 & 28.57977\end{array}$

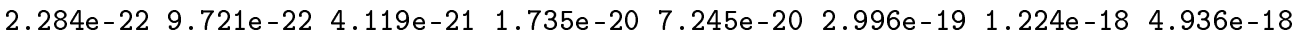

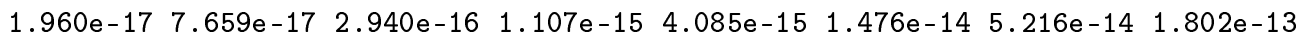
$6.079 e-13 \quad 2.002 e-12 \quad 6.433 e-12 \quad 2.016 e-11 \quad 6.159 e-11 \quad 1.834 e-10 \quad 5.321 e-10 \quad 1.504 e-09$ $\begin{array}{llllllll}4.144 e-09 & 1.112 e-08 & 2.908 e-08 & 7.407 e-08 & 1.837 e-07 & 4.433 e-07 & 1.039 e-06 & 2.357 e-06\end{array}$ 5.141e-06 $1.068 \mathrm{e}-05 \quad 2.092 \mathrm{e}-05 \quad 3.855 \mathrm{e}-05 \quad 6.736 \mathrm{e}-05 \quad 1.130 \mathrm{e}-04 \quad 1.829 \mathrm{e}-04 \quad 2.845 \mathrm{e}-04$

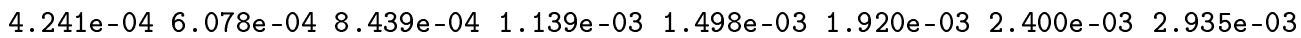
$3.517 e-03 \quad 4.141 e-03 \quad 4.805 e-03 \quad 5.486 e-03 \quad 6.157 e-03 \quad 6.758 e-03 \quad 7.206 e-03 \quad 7.445 e-03$

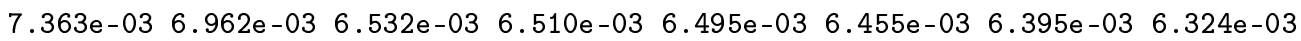
$\begin{array}{llllllll}5.977 e-03 & 5.892 e-03 & 5.776 e-03 & 5.632 e-03 & 5.463 e-03 & 4.647 e-03 & 3.758 e-03 & 2.890 e-03\end{array}$ $2.060 \mathrm{e}-03 \quad 1.224 \mathrm{e}-03 \quad 5.621 \mathrm{e}-04 \quad 1.944 \mathrm{e}-04 \quad 2.597 \mathrm{e}-05 \quad 9.259 \mathrm{e}-07 \quad 2.936 \mathrm{e}-08$

$\begin{array}{llllllll}7.400 e-43 & 4.444 e-42 & 2.709 e-41 & 1.675 e-40 & 1.048 e-39 & 6.640 e-39 & 4.250 e-38 & 2.747 e-37\end{array}$ $\begin{array}{lllllllll}1.791 e-36 & 1.178 e-35 & 7.802 e-35 & 5.204 e-34 & 3.494 e-33 & 2.359 e-32 & 1.599 e-31 & 1.091 e-30\end{array}$ $7.480 e-30 \quad 5.148 e-29 \quad 3.557 e-28 \quad 2.465 e-27 \quad 1.713 e-26 \quad 1.193 e-25 \quad 8.322 e-25 \quad 5.810 e-24$

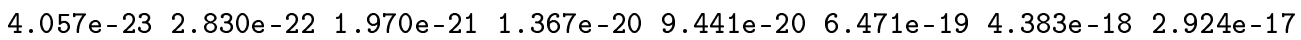
$1.902 e-16 \quad 1.191 e-15 \quad 7.093 e-15 \quad 3.991 e-14 \quad 2.136 e-13 \quad 1.099 e-12 \quad 5.458 e-12 \quad 2.598 e-11$ 1.179e-10 5.101e-10 2.111e-09 8.380e-09 $3.189 e-08 \quad 1.159 e-07 \quad 4.025 e-07 \quad 1.332 e-06$ 
$4.199 \mathrm{e}-06 \quad 1.262 \mathrm{e}-05 \quad 3.620 \mathrm{e}-05 \quad 9.919 \mathrm{e}-05 \quad 2.593 e-04 \quad 6.429 \mathrm{e}-04 \quad 1.497 \mathrm{e}-03 \quad 3.253 e-03$ $6.483 e-03 \quad 1.178 e-02 \quad 1.883 e-02 \quad 1.952 e-02 \quad 2.002 e-02 \quad 2.061 e-02 \quad 2.123 e-02 \quad 2.195 e-02$ $2.181 e-02 \quad 2.272 e-02 \quad 2.368 e-02 \quad 2.473 e-02 \quad 2.589 e-02 \quad 2.914 e-02 \quad 3.388 e-02 \quad 4.108 e-02$ $\begin{array}{lllllll}5.266 e-02 & 7.042 e-02 & 9.488 e-02 & 1.221 e-01 & 2.332 e-01 & 1.433 e-01 & 9.894 e-03\end{array}$

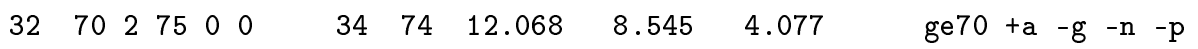
$\begin{array}{llllllll}0.65552 & 0.66404 & 0.67339 & 0.68362 & 0.69484 & 0.70713 & 0.72059 & 0.73534\end{array}$ $\begin{array}{lllllllll}0.75150 & 0.76921 & 0.78862 & 0.80988 & 0.83317 & 0.85870 & 0.88666 & 0.91731\end{array}$ $\begin{array}{llllllll}0.95088 & 0.98767 & 1.02797 & 1.07213 & 1.12052 & 1.17353 & 1.23162 & 1.29527\end{array}$ $\begin{array}{llllllll}1.36500 & 1.44141 & 1.52513 & 1.61686 & 1.71736 & 1.82748 & 1.94814 & 2.08034\end{array}$ $\begin{array}{llllllll}2.22519 & 2.38389 & 2.55779 & 2.74831 & 2.95707 & 3.18581 & 3.43642 & 3.71102\end{array}$ $\begin{array}{llllllll}4.01188 & 4.34154 & 4.70273 & 4.76759 & 4.89054 & 5.12364 & 5.56552 & 6.40321\end{array}$ $\begin{array}{llllllll}7.80068 & 7.91185 & 7.99125 & 8.01439 & 8.03991 & 8.06805 & 8.09910 & 8.13334\end{array}$ $\begin{array}{llllllll}8.17111 & 8.21276 & 8.25870 & 8.30937 & 8.36526 & 8.42690 & 8.49488 & 8.74379\end{array}$ $\begin{array}{llllllll}9.07774 & 9.52582 & 10.12700 & 10.93362 & 12.01588 & 13.46795 & 15.41621 & 18.03023\end{array}$ $21.53748 \quad 26.24321 \quad 30.24312$

$\begin{array}{lllllllllllllll}0.000 e+00 & 0.000 e+00 & 0.000 e+00 & 0.000 e+00 & 0.000 e+00 & 0.000 e+00 & 0.000 e+00 & 0.000 e+00\end{array}$ $\begin{array}{lllllllllllll}0.000 e+00 & 0.000 e+00 & 0.000 e+00 & 0.000 e+00 & 0.000 e+00 & 0.000 e+00 & 0.000 e+00 & 0.000 e+00\end{array}$ $0.000 e+00 \quad 8.548 e-35 \quad 9.326 e-34 \quad 1.091 e-32 \quad 1.361 e-31 \quad 1.798 e-30 \quad 2.496 e-29 \quad 3.614 e-28$ $5.414 e-27 \quad 8.311 e-26 \quad 1.299 e-24 \quad 2.048 e-23 \quad 3.227 e-22 \quad 5.039 e-21 \quad 7.736 e-201.157 e-18$ $\begin{array}{llllllll}1.676 e-17 & 2.332 e-16 & 3.097 e-15 & 3.900 e-14 & 4.624 e-13 & 5.144 e-12 & 5.338 e-11 & 5.139 e-10\end{array}$

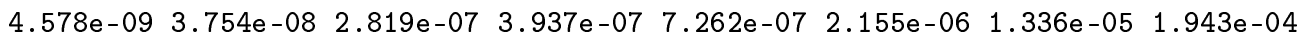
$2.062 e-03 \quad 1.878 e-03 \quad 2.004 e-03 \quad 1.920 e-03 \quad 1.776 e-03 \quad 1.652 e-03 \quad 1.539 e-03 \quad 1.451 e-03$ $\begin{array}{llllllll}1.326 e-03 & 1.223 e-03 & 1.150 e-03 & 1.073 e-03 & 1.024 e-03 & 9.863 e-04 & 9.686 e-04 & 9.189 e-04\end{array}$ $9.455 e-04 \quad 1.122 e-03 \quad 1.364 e-03 \quad 1.488 e-03 \quad 1.296 e-03 \quad 8.147 e-04 \quad 3.616 e-04 \quad 1.082 e-04$ $1.096 \mathrm{e}-052.904 \mathrm{e}-07 \quad 1.185 \mathrm{e}-08$ 


\section{Table II: Sample of Rate Tables}

\begin{tabular}{|c|c|c|c|c|c|c|c|}
\hline $\mathrm{Z} \quad \mathrm{A} \mathrm{p} \mathrm{b}$ & $\mathrm{c}$ & $\mathrm{x} e$ & f $h$ & & & & \\
\hline G1 & G2 & $\ldots$ G24 & & & & & \\
\hline $\mathrm{H} 1$ & $\mathrm{H} 2$ & . . H24 & & & & & \\
\hline ( R1 & $\mathrm{R} 2$ & ..R24 ) & & & & & \\
\hline ( U1 & $\mathrm{U} 2$ & ...U24 ) & & & & & \\
\hline$\cdot$ & & & & & & & \\
\hline 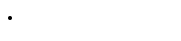 & & & & & & & \\
\hline & & & & & & & \\
\hline $\begin{array}{llll}31 & 70 & 0 & 1\end{array}$ & 1 & $70+n-g-$ & $-p-a$ & & & & \\
\hline $4.64 e+07$ & $4.54 \mathrm{e}+07$ & $4.50 e+07$ & $4.44 e+07$ & $4.41 e+07$ & $4.39 e+07$ & $4.39 e+07$ & $4.39 e+07$ \\
\hline $4.40 e+07$ & $4.42 e+07$ & $4.43 e+07$ & $4.52 e+07$ & $4.56 e+07$ & $4.54 e+07$ & $4.48 e+07$ & $4.40 e+07$ \\
\hline $4.29 e+07$ & $4.18 e+07$ & $4.06 e+07$ & $3.82 e+07$ & $3.60 e+07$ & $3.40 e+07$ & $3.21 \mathrm{e}+07$ & $3.04 e+07$ \\
\hline $4.64 e+07$ & $4.54 e+07$ & $4.50 e+07$ & $4.44 e+07$ & $4.41 e+07$ & $4.39 e+07$ & $4.39 e+07$ & $4.39 e+07$ \\
\hline $4.40 e+07$ & $4.41 \mathrm{e}+07$ & $4.43 e+07$ & $4.45 e+07$ & $4.29 e+07$ & $3.91 e+07$ & $3.43 e+07$ & $2.94 e+07$ \\
\hline $2.49 e+07$ & $2.10 e+07$ & $1.77 e+07$ & $1.25 e+07$ & $8.79 e+06$ & $6.12 e+06$ & $4.22 e+06$ & $2.89 e+06$ \\
\hline $8.53 e+04$ & $7.95 e+04$ & $7.69 e+04$ & $7.50 e+04$ & $7.55 e+04$ & $7.77 e+04$ & $8.12 e+04$ & $8.59 e+04$ \\
\hline $9.18 e+04$ & $9.88 e+04$ & $1.07 e+05$ & $1.62 \mathrm{e}+05$ & $2.39 e+05$ & $3.32 e+05$ & $4.41 e+05$ & $5.62 e+05$ \\
\hline $6.94 e+05$ & $8.34 e+05$ & $9.80 e+05$ & $1.29 e+06$ & $1.60 e+06$ & $1.92 e+06$ & $2.24 e+06$ & $2.56 e+06$ \\
\hline $8.53 e+04$ & $7.95 e+04$ & $7.69 e+04$ & $7.50 e+04$ & $7.55 e+04$ & $7.77 e+04$ & $8.12 e+04$ & $8.60 e+04$ \\
\hline $9.20 e+04$ & $9.93 e+04$ & $1.08 e+05$ & $1.74 \mathrm{e}+05$ & $2.77 e+05$ & $4.09 e+05$ & $5.54 e+05$ & $7.06 e+05$ \\
\hline $8.60 e+05$ & $1.02 e+06$ & $1.18 e+06$ & $1.54 e+06$ & $2.01 e+06$ & $2.69 e+06$ & $3.71 e+06$ & $5.16 e+06$ \\
\hline $1.57 e+03$ & $1.59 e+03$ & $1.63 e+03$ & $1.73 e+03$ & $1.83 e+03$ & $1.95 e+03$ & $2.10 e+03$ & $2.26 \mathrm{e}+03$ \\
\hline $2.45 e+03$ & $2.66 e+03$ & $2.90 \mathrm{e}+03$ & $4.45 e+03$ & $6.66 \mathrm{e}+03$ & $9.75 e+03$ & $1.40 \mathrm{e}+04$ & $2.00 e+04$ \\
\hline $2.81 e+04$ & $3.89 e+04$ & $5.32 e+04$ & $9.40 e+04$ & $1.55 e+05$ & $2.38 e+05$ & $3.45 e+05$ & $4.78 e+05$ \\
\hline $1.57 e+03$ & $1.59 e+03$ & $1.63 e+03$ & $1.73 e+03$ & $1.83 e+03$ & $1.95 e+03$ & $2.10 e+03$ & $2.27 e+03$ \\
\hline $2.46 e+03$ & $2.70 e+03$ & $2.97 e+03$ & $5.34 e+03$ & $1.07 e+04$ & $2.14 e+04$ & $4.06 e+04$ & $7.28 e+04$ \\
\hline $1.24 \mathrm{e}+05$ & $2.00 e+05$ & $3.11 \mathrm{e}+05$ & $6.78 e+05$ & $1.33 e+06$ & $2.39 e+06$ & $4.01 e+06$ & $6.33 e+06$ \\
\hline $\begin{array}{llll}31 & 70 & 1 & 1\end{array}$ & 1 & $70+p-g-$ & $-\mathrm{n}-\mathrm{a}$ & & & & \\
\hline $4.69 e-23$ & $2.93 e-18$ & $2.77 e-15$ & $1.43 e-11$ & $3.07 e-09$ & $1.36 e-07$ & $2.38 e-06$ & $2.32 e-05$ \\
\hline $1.50 e-04$ & $7.17 e-04$ & $2.74 e-03$ & $2.93 e-01$ & $5.21 e+00$ & $3.81 e+01$ & $1.65 e+02$ & $5.10 e+02$ \\
\hline $1.25 e+03$ & $2.58 e+03$ & $4.72 e+03$ & $1.21 e+04$ & $2.45 e+04$ & $4.22 e+04$ & $6.50 e+04$ & $9.24 e+04$ \\
\hline $4.69 e-23$ & $2.93 e-18$ & $2.77 e-15$ & $1.43 e-11$ & $3.07 e-09$ & e-07 & $2.38 e-06$ & $2.32 e-05$ \\
\hline $1.50 e-04$ & $7.17 e-04$ & $2.74 e-03$ & $2.94 e-01$ & $5.30 e+00$ & $3.85 e+01$ & $1.60 \mathrm{e}+02$ & $4.62 e+02$ \\
\hline $1.04 e+03$ & $1.97 e+03$ & $3.27 e+03$ & $6.85 e+03$ & $1.10 e+04$ & $1.48 e+04$ & $1.73 e+04$ & $1.82 e+04$ \\
\hline $3.94 e-22$ & $2.96 e-17$ & $3.27 e-14$ & $2.11 \mathrm{e}-10$ & $5.27 e-08$ & $2.65 e-06$ & $5.22 e-05$ & $5.61 e-04$ \\
\hline $3.96 e-03$ & $2.06 e-02$ & $8.46 e-02$ & $1.18 e+01$ & $2.48 e+02$ & $2.02 e+03$ & $9.56 e+03$ & $3.18 e+04$ \\
\hline $8.32 e+04$ & $1.83 e+05$ & $3.56 e+05$ & $1.02 e+06$ & $2.28 e+06$ & $4.32 e+06$ & $7.27 e+06$ & $1.12 \mathrm{e}+07$ \\
\hline $3.94 e-22$ & $2.96 e-17$ & $3.27 e-14$ & $2.11 \mathrm{e}-10$ & $5.27 e-08$ & $2.65 e-06$ & $5.22 e-05$ & $5.61 \mathrm{e}-04$ \\
\hline $3.96 e-03$ & $2.06 e-02$ & $8.46 e-02$ & $1.18 e+01$ & $2.48 e+02$ & $2.02 e+03$ & $9.55 e+03$ & $3.17 e+04$ \\
\hline $8.30 e+04$ & $1.83 e+05$ & $3.53 e+05$ & $1.01 e+06$ & $2.23 e+06$ & $4.18 e+06$ & $6.94 e+06$ & $1.05 e+07$ \\
\hline
\end{tabular}




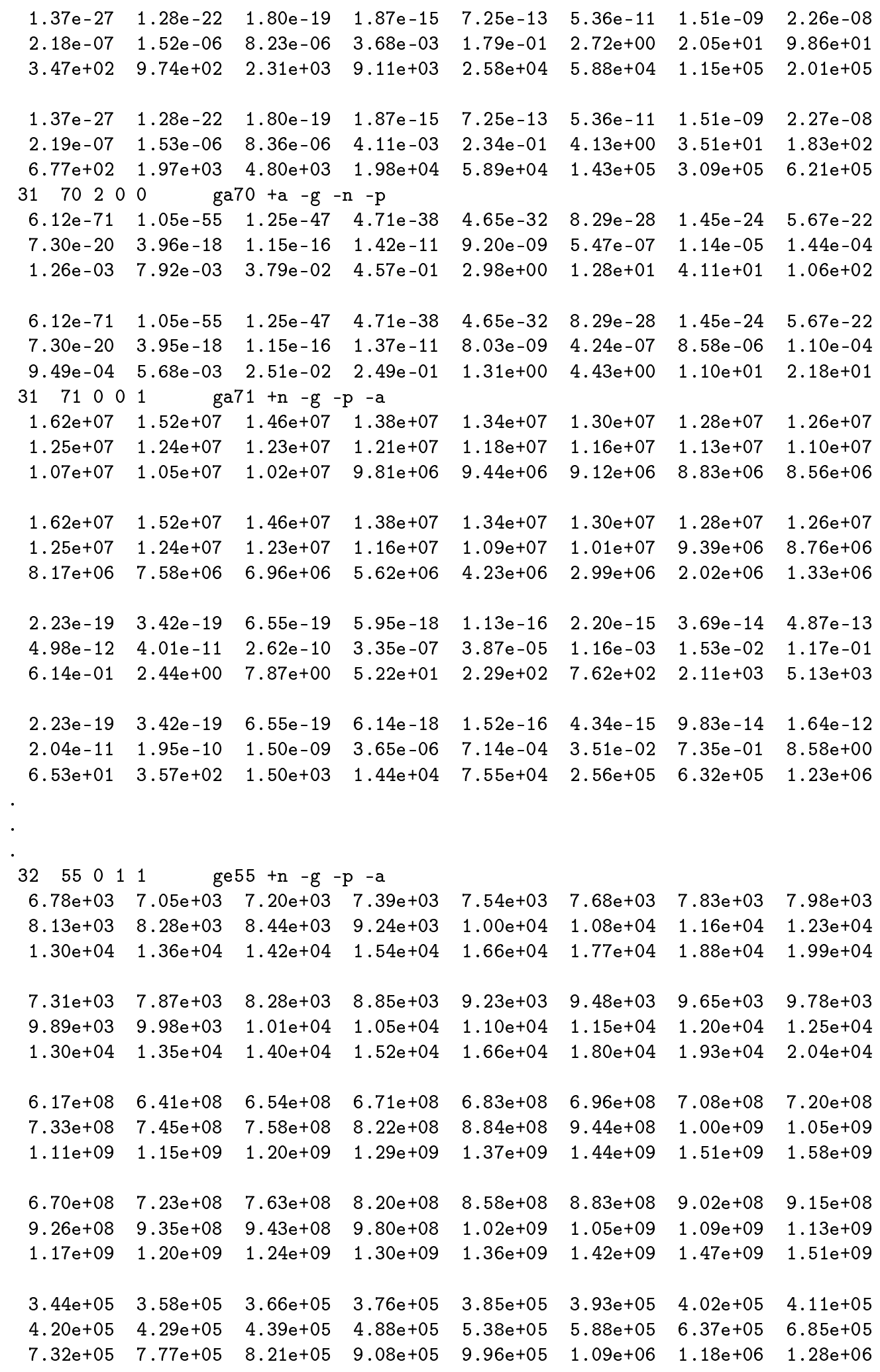




\begin{tabular}{|c|c|c|c|c|c|c|c|}
\hline $3.70 e+05$ & $3.99 e+05$ & $4.20 \mathrm{e}+05$ & $4.51 e+05$ & $4.71 e+05$ & $4.85 e+05$ & $4.96 e+05$ & $5.05 e+05$ \\
\hline $5.12 e+05$ & $5.19 e+05$ & $5.26 e+05$ & $5.63 e+05$ & $6.03 e+05$ & $6.46 e+05$ & $6.90 e+05$ & $7.35 e+05$ \\
\hline \multicolumn{8}{|c|}{ 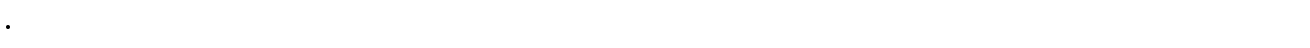 } \\
\hline \\
\hline \multirow{2}{*}{\multicolumn{8}{|c|}{3}} \\
\hline & & & & & & & \\
\hline $1.66 \mathrm{e}+07$ & $1.61 e+07$ & $1.58 e+07$ & $1.57 e+07$ & $1.56 e+07$ & $1.57 e+07$ & $1.58 e+07$ & $1.60 e+07$ \\
\hline $1.62 e+07$ & $1.64 e+07$ & $1.66 e+07$ & $1.80 e+07$ & $1.94 e+07$ & $2.08 e+07$ & $2.20 e+07$ & $2.30 e+07$ \\
\hline $2.39 e+07$ & $2.46 e+07$ & $2.52 e+07$ & $2.62 e+07$ & $2.69 e+07$ & $2.75 e+07$ & $2.78 e+07$ & $2.81 e+07$ \\
\hline $1.66 e+07$ & $1.61 e+07$ & $1.58 e+07$ & $1.57 e+07$ & $1.56 e+07$ & $1.57 e+07$ & $1.58 e+07$ & $1.60 \mathrm{e}+07$ \\
\hline $1.62 \mathrm{e}+07$ & $1.64 \mathrm{e}+07$ & $1.66 e+07$ & $1.80 e+07$ & $1.96 e+07$ & $2.13 e+07$ & $2.29 e+07$ & $2.42 e+07$ \\
\hline $2.53 e+07$ & $2.59 e+07$ & $2.59 e+07$ & $2.40 e+07$ & $2.01 e+07$ & $1.52 \mathrm{e}+07$ & $1.06 e+07$ & $6.90 e+06$ \\
\hline $1.24 \mathrm{e}-01$ & $1.32 e-01$ & $1.41 \mathrm{e}-01$ & $1.67 e-01$ & $2.01 e-01$ & $2.49 e-01$ & $3.16 e-01$ & $4.11 e-01$ \\
\hline $5.51 e-01$ & $7.54 e-01$ & $1.05 e+00$ & $5.77 e+00$ & $2.88 e+01$ & $1.24 \mathrm{e}+02$ & $4.55 e+02$ & $1.41 e+03$ \\
\hline $3.73 e+03$ & $8.65 e+03$ & $1.79 e+04$ & $5.93 e+04$ & $1.50 e+05$ & $3.17 e+05$ & $5.82 e+05$ & $9.64 e+05$ \\
\hline $1.24 e-01$ & $1.32 e-01$ & $1.41 \mathrm{e}-01$ & $1.67 e-01$ & $2.01 e-01$ & $2.49 e-01$ & $3.16 e-01$ & $4.12 e-01$ \\
\hline $5.52 e-01$ & $7.63 e-01$ & $1.09 e+00$ & $9.52 e+00$ & $9.50 e+01$ & $7.32 e+02$ & $4.23 e+03$ & $1.88 e+04$ \\
\hline $6.72 e+04$ & $1.98 e+05$ & $4.97 e+05$ & $2.14 e+06$ & $6.22 e+06$ & $1.38 e+07$ & $2.52 e+07$ & $4.01 e+07$ \\
\hline $\begin{array}{llll}32 & 70 & 1 & 0\end{array}$ & 1 & $70+p-g-$ & $\mathrm{n}-\mathrm{a}$ & & & & \\
\hline $7.61 e-23$ & $7.14 e-18$ & $9.14 e-15$ & $7.11 \mathrm{e}-11$ & $2.01 e-08$ & $1.10 e-06$ & $2.32 e-05$ & $2.61 e-04$ \\
\hline $1.90 e-03$ & $9.95 e-03$ & $4.07 e-02$ & $4.80 e+00$ & $7.80 e+01$ & $5.00 e+02$ & $1.92 e+03$ & $5.34 e+03$ \\
\hline $1.20 \mathrm{e}+04$ & $2.33 e+04$ & $4.04 e+04$ & $9.64 e+04$ & $1.86 e+05$ & $3.11 e+05$ & $4.70 e+05$ & $6.59 e+05$ \\
\hline $7.61 e-23$ & $7.14 e-18$ & $9.14 e-15$ & $7.11 e-11$ & $2.01 e-08$ & $1.10 e-06$ & $2.32 e-05$ & $2.61 e-04$ \\
\hline $1.90 e-03$ & $9.95 e-03$ & $4.07 e-02$ & $4.80 e+00$ & $7.79 e+01$ & $5.01 e+02$ & $1.92 e+03$ & $5.35 e+03$ \\
\hline $1.20 e+04$ & $2.28 e+04$ & $3.84 e+04$ & $8.10 e+04$ & $1.25 e+05$ & $1.51 e+05$ & $1.50 e+05$ & $1.29 e+05$ \\
\hline $3.34 e-42$ & $7.00 e-36$ & $1.36 e-31$ & $9.30 e-26$ & $8.93 e-22$ & $8.02 e-19$ & $1.56 e-16$ & $1.08 e-14$ \\
\hline $3.60 e-13$ & $7.02 e-12$ & $9.11 e-11$ & $7.75 e-07$ & $2.23 e-04$ & $1.19 e-02$ & $2.32 e-01$ & $2.37 e+00$ \\
\hline $1.53 e+01$ & $7.05 e+01$ & $2.53 e+02$ & $1.88 e+03$ & $8.45 e+03$ & $2.72 e+04$ & $6.93 e+04$ & $1.49 e+05$ \\
\hline $3.34 e-42$ & $7.00 e-36$ & $1.36 e-31$ & $9.30 e-26$ & $8.93 e-22$ & $8.02 e-19$ & $1.56 e-16$ & $1.08 e-14$ \\
\hline $3.62 e-13$ & $7.11 e-12$ & $9.39 e-11$ & $1.02 e-06$ & $4.28 e-04$ & $3.26 e-02$ & $8.75 e-01$ & $1.17 e+01$ \\
\hline $9.34 e+01$ & $5.11 e+02$ & $2.07 e+03$ & $1.77 e+04$ & $8.19 e+04$ & $2.55 e+05$ & $6.11 \mathrm{e}+05$ & $1.21 e+06$ \\
\hline $\begin{array}{llll}32 & 70 & 2 & 0\end{array}$ & 0 & $70+a-g-$ & $n-p$ & & & & \\
\hline $3.70 e-74$ & $1.33 e-57$ & $5.31 e-49$ & $3.86 e-39$ & $5.15 e-33$ & $1.13 e-28$ & $2.26 e-25$ & $9.58 e-23$ \\
\hline $1.38 e-20$ & $9.11 e-19$ & $3.31 e-17$ & $9.48 e-12$ & $2.29 e-08$ & $5.01 e-06$ & $2.56 e-04$ & $5.03 e-03$ \\
\hline $5.12 e-02$ & $3.28 e-01$ & $1.50 e+00$ & $1.57 e+01$ & $8.73 e+01$ & $3.23 e+02$ & $9.01 e+02$ & $2.05 e+03$ \\
\hline $3.70 e-74$ & $1.33 e-57$ & $5.31 e-49$ & $3.86 e-39$ & $5.15 e-33$ & $1.13 e-28$ & $2.26 e-25$ & $9.58 e-23$ \\
\hline $1.38 e-20$ & $9.11 e-19$ & $3.31 e-17$ & $9.48 e-12$ & $2.29 e-08$ & $4.98 e-06$ & $2.48 e-04$ & $4.64 e-03$ \\
\hline $4.40 e-02$ & $2.56 e-01$ & $1.04 e+00$ & $7.79 e+00$ & $2.81 e+01$ & $6.19 e+01$ & $9.75 e+01$ & $1.22 \mathrm{e}+02$ \\
\hline
\end{tabular}




\section{Table III: Sample of Nuclear Level Table}

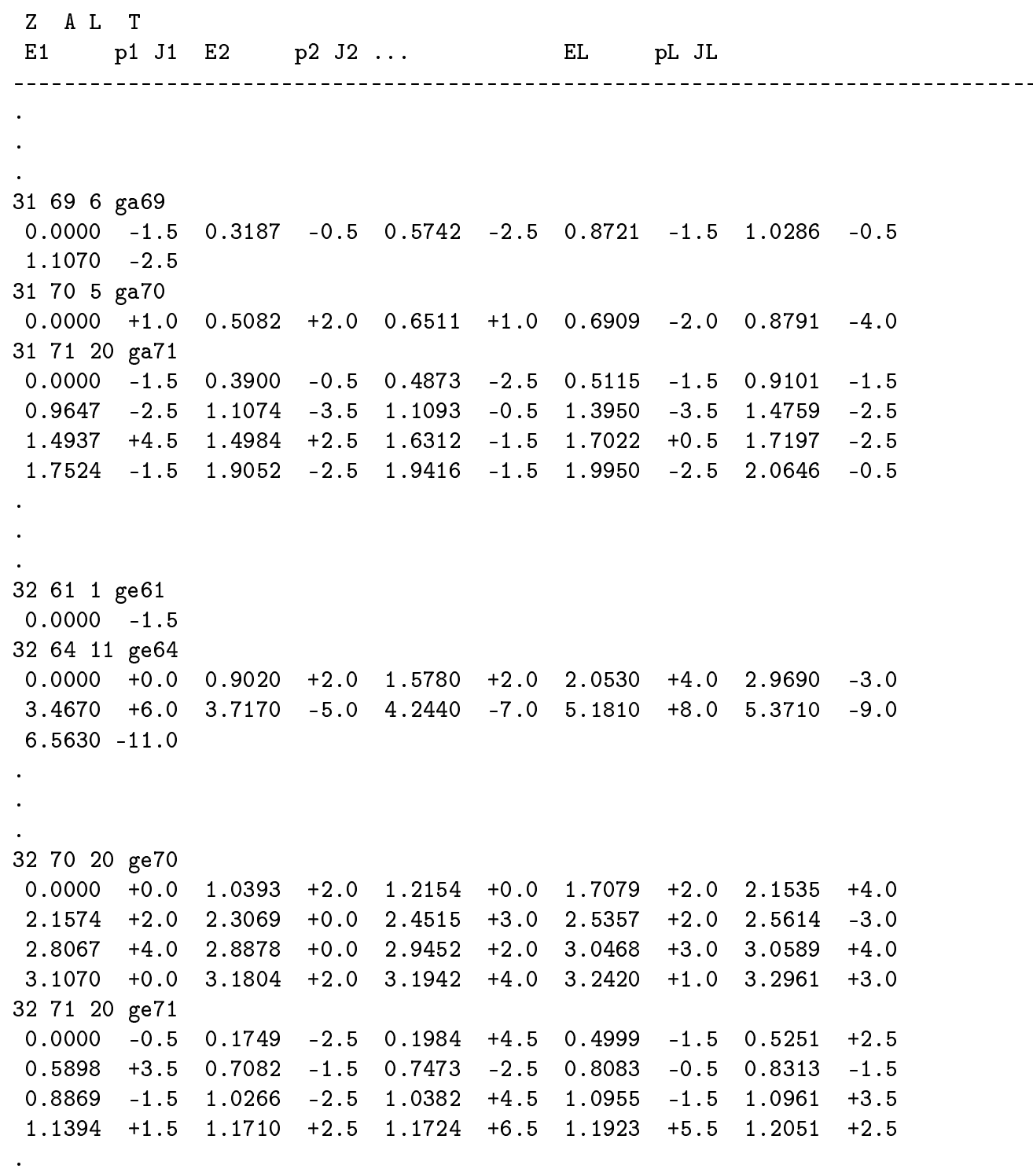

